\title{
Electron Cyclotron Heating in RFP plasmas
}

\author{
R. Bilato*, F. Volpe ${ }^{\dagger}$, E. Poli*, A. Köhn**, R. Cavazzana ${ }^{\ddagger}$, R. Paccagnella \\ and D. Farina ${ }^{\S}$
}
* MPI für Plasmaphysik - Euratom Association Boltzmannstr. 2, D-85748 Garching, Germany
${ }^{\dagger}$ Department of Engineering Physics, University of Wisconsin, Madison, WI, USA
** Institut für Plasmaforschung, Universität Stuttgart - Stuttgart, Germany
$¥$ Consorzio RFX - Associazione EURATOM-ENEA sulla fusione - Padova, Italy
${ }^{\S}$ IFP-CNR, EURATOM-ENEA-CNR Association - Milano, Italy

\begin{abstract}
Reversed field pinches (RFP) plasmas are typically overdense $\left(\omega_{\text {pe }}>\Omega_{\text {ce }}\right)$ and thus not suitable for conventional electron cyclotron (EC) heating and current drive. In recent high plasma current discharges $\left(\mathrm{I}_{\mathrm{p}}>1.5 \mathrm{MA}\right)$, however, the RFX-mod device was operated in underdense conditions $\left(\omega_{\mathrm{pe}}<\Omega_{\mathrm{ce}}\right)$ for the first time in an RFP. Thus, it is now possible to envisage heating the RFP plasma core by conventional EC at the 2nd harmonic, in the ordinary or extraordinary mode. We present a preliminary study of EC-heating feasibility in RFX-mod with the use of beamtracing and full-wave codes. Although not competitive - as a heating system - with multi-MW Ohmic heating in an RFP, EC might be useful for perturbative transport studies, even at moderate power (hundreds of $\mathrm{kW}$ ), and, more generally, for applications requiring localized power deposition.
\end{abstract}

Keywords: ECRH, RFP, beam-tracing, full-wave code

PACS: 42.15.Dp, 52.35.Hr, 52.40.Db, 52.50.Sw, 52.55.Hc

1 - Introduction. There is an ongoing interest in using radio-frequency (rf) waves in RFPs in order to control the current density profile in the plasma periphery. Since RFPs typically operate with overdense plasmas $\left(\omega_{\mathrm{pe}} / \Omega_{\mathrm{ce}} \gg 1\right.$, with $\omega_{\mathrm{pe}}=\left(e^{2} n_{e} /\left(\varepsilon_{0} m_{e}\right)\right)^{1 / 2}$ the electron plasma angular frequency and $\Omega_{\mathrm{ce}}=e B / m_{e}$ the electron cyclotron angular frequency), except for a thin layer at the edge, the efforts have been oriented to lower hybrid and electron Bernstein waves [1, 2]. However, none of the usual rf schemes can access the plasma core of typical RFP plasmas, except for high plasma current discharges $\left(I_{p}>1.5 \mathrm{MA}\right)$ recently obtained in the RFX-mod device with an extended set of active control coils [3].

The confining magnetic field on axis scales with the plasma current $I_{p}$ like $B_{0[\mathrm{~T}]} \approx$ $\delta_{d} \cdot \mu_{0} I_{p} / 2 \pi a=0.44 \delta_{d} I_{p[\mathrm{MA}]}$, with $a=0.46 \mathrm{~m}$ the RFX-mod minor radius and $\delta_{d}$ the RFP paramagnetic amplification factor. According to the $\mu \& p$ model (see Eq.(6) of [2] for a simplified version), for typical RFX-mod equilibria $2.2<\delta_{d}<2$.6. Hereafter, we assume $\delta_{d}=2.3$, which implies $B_{0[\mathrm{~T}]} \approx I_{p[\mathrm{MA}]}$. Consequently, the nth EC harmonic frequency on the plasma axis scales with $I_{p}$ like $f_{[\mathrm{GHz}]}=28 n I_{p[\mathrm{MA}]}$. For $I_{p}>1.5 \mathrm{MA}$ the 2 nd EC (i.e. $n=2$ ) harmonic in the plasma core is above $80 \mathrm{GHz}$. At this range of frequencies, the wave accessibility constraint on the plasma density is not particularly severe. In fact, if we make use of the dimensionless density $X=\omega_{\mathrm{pe}}^{2} / \omega^{2}$ and magnetic field $Y=\Omega_{\mathrm{ce}} / \omega$, where $\omega=2 \pi f$ is the angular frequency of the injected waves, the cutoffs of O- and X-mode are $X=1$ and $X=(1-Y)\left(1-N_{\|}^{2}\right)=(n-1) / n(1-$ $\left.N_{\|}^{2}\right)$, respectively, with $N_{\|}$the parallel component of the refractive index. Hereafter we 
consider almost perpendicular incidence, i.e. $N_{\|} \approx 0$. The $\mathrm{O}$ - and $\mathrm{X}$-mode can be rewritten as $n_{e\left[10^{19} \mathrm{~m}^{-3}\right]}=n^{2} I_{p[\mathrm{MA}]}^{2}$ and $n_{e\left[10^{19} \mathrm{~m}^{-3}\right]}=n(n-1) I_{p[\mathrm{MA}]}^{2}$. Table (1) shows the values of the cutoff densities for two suitable commercial frequencies together with the corresponding minimum $I_{p}$ values in order to have the 2 nd harmonic in the plasma center. We also report the corresponding values of the operation parameter $I_{p} / N$, i.e. the ratio between the plasma current and the line-averaged density $N=\pi a^{2}<n>: I_{p} / N$ amounts to the ratio of Greenwald limit to the line density $\langle n\rangle$. In the following, we consider $84 \mathrm{GHz}$, a compromise between the density limit and RFX-mod operation

\begin{tabular}{|l|c|cc|cc|}
\hline $\mathrm{f}_{[\mathrm{GHz}]}$ & $\mathrm{I}_{\mathrm{p}[\mathrm{MA}]}$ & \multicolumn{2}{|c|}{$n_{e 0\left[10^{19} \mathrm{~m}^{-3}\right]}$} & \multicolumn{2}{|c|}{$I_{p} / N_{\left[10^{-14} \mathrm{Am}\right]}$} \\
\hline & & $O$ & $X$ & $O$ & $X$ \\
\hline 84 & 1.5 & 9 & 4.5 & 3 & 6 \\
\hline 95 & 1.7 & 12 & 6 & 2.5 & 5 \\
\hline
\end{tabular}

Table 1: Cutoff densities for the $O$ and $X 2$ schemes.

space. At this frequency, $I_{p}$ can range from about $1.5 \mathrm{MA}\left(2 \Omega_{\mathrm{ce}}\right.$ on axis) up to the maximum forseen $2 \mathrm{MA}$ ( $2 \Omega_{\mathrm{ce}}$ at about half of the minor radius), and beyond. However, for a given frequency, higher $I_{p}$ values have lower cutoff densities. This is partially compensated by the fact that by increasing $I_{p}$ the 2 nd EC resonance moves towards the plasma periphery, where the density decreases. To access higher densities, one has to launch the O-mode, which has a much higher density limit. However, O2 scheme suffers form a very low single-pass absorption.

One peculiar feature of RFP plasmas is the almost poloidally symmetric confining magnetic field, which means almost poloidal EC resonance surface, as shown in Fig (1). As a consequence, the radial position of the hf-power deposition pro- $\underline{E}$ file cannot be controlled with the vertical $N$ launch angle, as in tokamaks. Moreover, the EC resonance is reachable only for a limited range of the vertical tilt angle, i.e. too tilted beams miss the $\mathrm{EC}$ resonance. On the other hand, the beam along its single transit encounters the EC resonance twice, increasing the single-pass absorption, particularly favourable for the O2. Concerning X2 scheme, if the wave encounters the EC resonance before the X-mode cutoff, the absorption of the X-mode is almost complete.

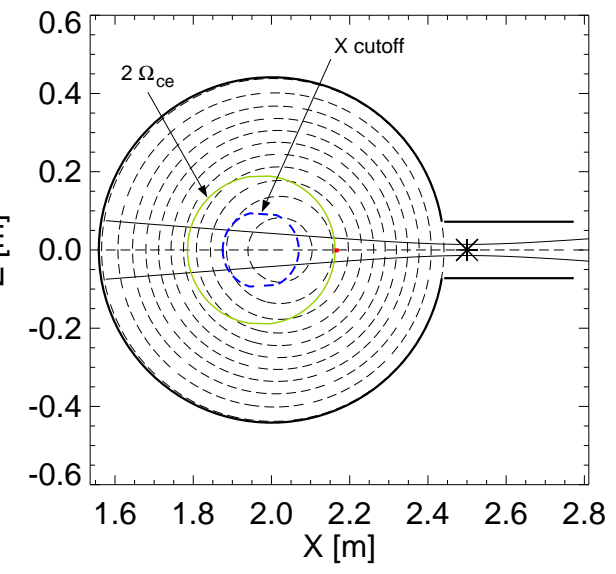

Figure 1: Beam launch geometry in RFX-mod. The dotted lines are the magnetic flux surfaces. The star shows the position of the waist. In the next section we discuss the EC launch constraints in RFX-mod and the X2 absorption for three plasma currents. We also evaluate the single pass absorption of $\mathrm{O} 2$ mode. In the last section, we address the issue of how much power one needs to have measurable effects on the electron temperature.

2 - Launch geometry and EC absorption in RFX-mod. The largest ports in RFX-mod for EC access are the equatorial pumping ports. Because of the lack of space inside the vessel, the control of the beam incidence angles must be done remotely. Fig.(1) shows the direct way of externally controlling the beam. 
The maximum tilting angle and the beam focusing are constrained mainly by the diameter and length of the cylindrical ports. Fig. (2) shows that the range of angular adjustment in the vertical direction $\alpha$ (and, by symmetry, in the horizontal direction as well, $\beta$ ) decreases when the beam waist is increased from $w_{0}=3 \lambda$ to $5 \lambda$, with $\lambda=c / v$ the vacuum wavelength. On the other hand a narrow waist $w_{0}$ implies more rapid beam divergence. A fair compromise is $w_{0}=4 \lambda$ and the waist slightly inside the pumping port $\left(x_{w}=55 \mathrm{~cm}\right)$. Alternatively, it might be possible to use the remotely steering system initially designed for ITER [4]. We calculate the wave propagation and absorption with the beam tracing TORBEAM code [5]. For the density profile we assume $n_{\mathrm{e}}(x)=$ $n_{\mathrm{e} 0}\left(1-x^{6}\right)+n_{\text {edge }}$ with $<n>=0.86 \cdot n_{\mathrm{e} 0}$

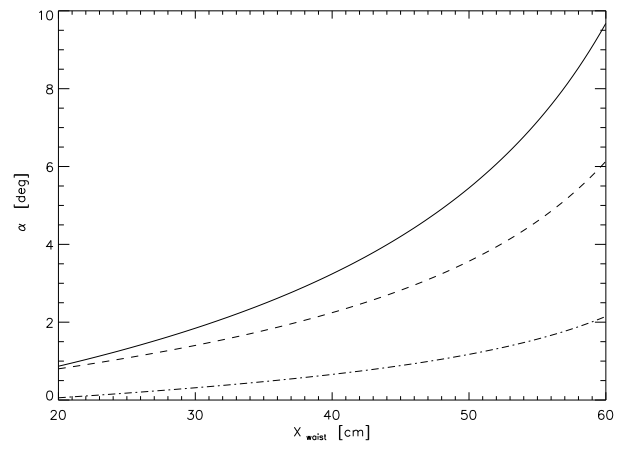

Figure 2: Maximum tilting angle as function of $X_{w}$ for $w_{0}=3$ (solid), 4 (dashed), 5 (dotdashed) $\lambda$ waists.

and $n_{\text {edge }} / n_{\mathrm{e} 0}=0.05$. The electron temperature profile is $T_{\mathrm{e} 0}(x)=T_{0}\left(1-x^{3}\right)+T_{\text {edge }}$, with $T_{0}=1.2 \mathrm{keV}$ and $T_{\text {edge }} / T_{0}=0.05$. We consider three plasma currents with the same
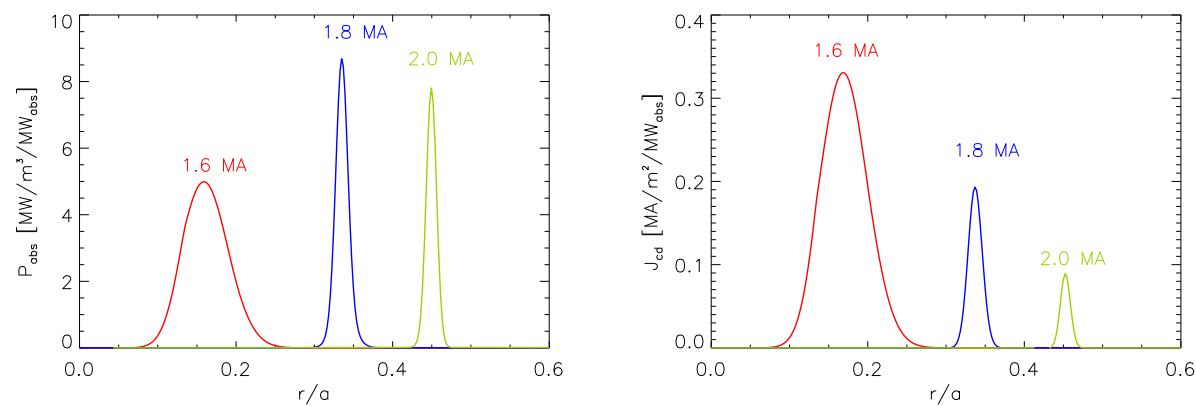

Figure 3: X2 scheme: EC deposited power (a) and driven current (b) profiles in correspondence of the optimal angles for highest power absorption (a) and driven current (b) peaks.

density profile and density on axis $\left(n_{\mathrm{e} 0}=4 \cdot 10^{19} \mathrm{~m}^{-3}\right)$, precisely $I_{p}=1.6,1.8,2.0 \mathrm{MA}$ with $I_{p} / N=6.5,7.3,8.1$ respectively. For all these currents we have done a scan in poloidal and toroidal launch angles to find the optimal absorbed power conditions for the $\mathrm{X} 2$ scheme. There is a wide range of launch angles around the perpendicular injection with complete power absorption. Fig. (3.a) shows the absorbed power density profiles in correspondence of launch angles with the highest absorption peak. These values are for $1 \mathrm{MW}$ of absorbed power and they scale linearly with the total absorbed power. The width of the deposition profile depends mainly on the divergence of the beam which increases towards the plasma center (see Fig. (1)). The height depends both on the width and on the specific volume around the resonance, which increases with increasing $x$. Fig. (3.b) shows the EC driven current density profiles for the optimal launch angles, which are not the same for the three cases. Also due to the almost perpendicular launch (not optimal for CD), the EC driven current densities are much smaller than the corresponding local plasma poloidal and toroidal current densities. 
Finally, in optimized scenarios with $I_{p} / N<6$, the same system can be used to launch the $\mathrm{O}$ mode. Fig. (4) shows the EC singlepass as function of the launching angles for $I_{p}=1.8 \mathrm{MA}$, and $I_{p} / N=6$. Because of the low electron temperature and the relative high densities, the single-pass absorption for the O-mode is less than $30 \%$, and for injection angles beyond the limit reachable with the direct launch system. It might be that multi-reflections from the graphite wall increase the total fraction of the absorbed power. However, in order to reach the angles of highest driven currents and of best $\mathrm{O}$-mode absorption, it is necessary to foresee a remotely steering system.

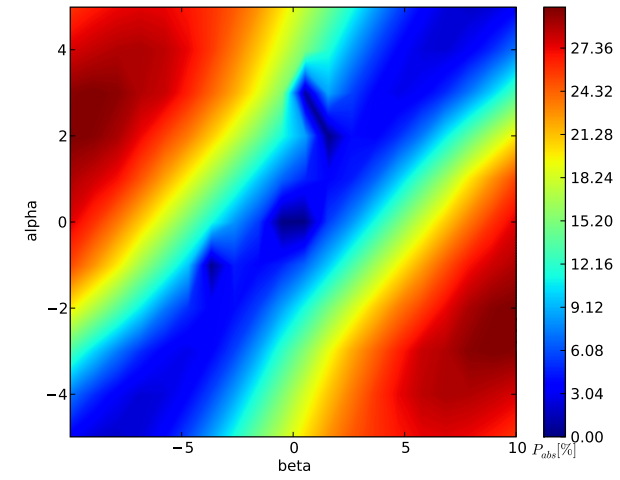

Figure 4: Single pass absorption for $\mathrm{O} 2$ scheme.

3 - Discussion. Finally, we compare the EC deposited power density with the local Ohmic one. The latter is estimated with the neoclassical Spitzer resistivity, corrected so as to have the total Ohmic power equal to the V loop $(\approx 20$ Volt $)$ times the plasma current. From this analysis, the local Ohmic power density at the maxima of the EC deposition profiles for the three plasma currents are respectively 3.8, 4.5, and 5.0 and $\mathrm{MW} / \mathrm{m}^{3}$. These values are comparable with the local maximum EC absorbed power density already for $500 \mathrm{~kW}$ of absorbed power. In addition, to quantify the effects of EC heating on $T_{e}$, we consider a simplified version of the heat diffusion equation,

$$
\frac{\partial T_{e}(x, t)}{\partial t}=\frac{1}{x} \frac{\partial}{\partial x}\left[x \chi(x) \frac{\partial T_{e}(x, t)}{\partial x}\right]+\left[\frac{T_{\mathrm{e} 0}(x)}{T_{e}(x, t)}\right]^{3 / 2} P_{\mathrm{Ohm}}(x)+P_{\mathrm{EC}}(x, t)
$$

where the density is assumed to evolve on a much longer time scale than the temperature. The diffusion coefficient $\chi$ is evaluated from the steady-state solution for $T_{\mathrm{e}}=T_{\mathrm{e} 0}(x)$ and with only Ohmic heating. The temperature dependence of resistivity is taken into account with the factor in front of $P_{\mathrm{Ohm}}$. According to this simplified model, for $1 \mathrm{MW}$ of EC coupled power the variations of the $T_{e}$ on axis are about $5 \%$. This rough analysis shows that EC heating might be suited for studies of RFX-mod transport, but less for additional heating.

Acknowledgments - we are indebted to S. Ortolani and M. Valisa for many helpful discussions, and to Y. Turkin for the help with the numerical equilibria format.

\section{REFERENCES}

1. FOREST, C., HARVEY, R., and SMIRNOV, A., Nuclear Fusion 41 (2001) 619.

2. BILATO, R., VOLPE, F., KOHN, A., et al., Nuclear Fusion 49 (2009) 075020.

3. PACCAGNElla, R., ORTOLANI, S., ZANCA, P., et al., Physical Review Letters 97 (2006) 075001.

4. VERHOEVEN, A. G. A., ELZENDOORN, B. S. Q., BONGERS, W. A., et al., Journal of Physics: Conference Series 25 (2005) 84.

5. POli, E., PEEters, A. G., and PEREVErZEV, G. V., Comp. Phys. Comm. 136 (2001) 90. 